\title{
OPTIMAL NOISE LEVEL FOR IMPERCEPTIBLE VIBROTACTILE STIMULATION DURING A FORCE STABILITY TASK
}

\author{
Courtney A. Haynes ${ }^{1}$, Matthew S. Tenan ${ }^{2}$, Antony D. Passaro ${ }^{3}$, Andrew J. Tweedell ${ }^{1}$ \\ ${ }^{1}$ U.S. Army Research Laboratory, Human Research and Engineering Directorate, Aberdeen \\ Proving Ground, MD, USA \\ ${ }^{2}$ U.S. Army Research Laboratory, Human Research and Engineering Directorate, Research \\ Triangle Park, Durham, NC, USA \\ ${ }^{3}$ U.S. Army Research Laboratory, Human Research and Engineering Directorate, ARL West, \\ Playa Vista, CA, USA \\ Corresponding author email: courtney.a.haynes2.civ@mail.mil
}

\begin{abstract}
Imperceptible vibratory noise stimulation has shown to be an effective means of improving stability for both whole body postural control and simple motor control tasks. While the physiological mechanism affording this improvement is uncertain, it is suspected that sensory noise stimulation may elicit a stochastic resonance-like effect within the somatosensory system. A stochastic resonance effect describes the phenomenon in which noise added to a non-linear system improves signal detection rather than degrading it. One hallmark of stochastic resonance is the existence of an optimal noise level which elicits the best system performance. There is disagreement in the literature regarding the presence of an optimal stimulation level for motor stability in humans. The goals of this study were to: 1) determine optimal stimulation level as a function of an individual's sub-sensory threshold level, and 2) to determine whether performance of a force stability task was significantly better when subjects received stimulation at this identified optimal level compared to other sub-sensory threshold stimulation levels. Eighteen (18) participants completed an isometric finger flexion task with visual feedback while receiving noise stimulation scaled to varying percentages of their individual sub-sensory threshold level. Performance for this force stabilization task was quantified as the root-mean-square (RMS) error between the target force and the actual generated force values. Despite controlling all other signal properties and varying only amplitude, optimal noise stimulation values still varied widely across participants (10-100\% sub-sensory threshold level). Statistical modeling revealed a significant improvement in task performance with optimal noise stimulation compared to other sub-sensory stimulation levels $(\mathrm{p} \leq 0.019)$ with estimated marginal mean differences in force errors ranging from 0.13 to $0.23 \mathrm{~N}$. Moderate significant Spearman correlations $\left(\mathrm{r}_{\mathrm{s}}=0.49\right.$ and $r_{s}=0.56$, respectively) were found between finger flexion maximal voluntary contraction (MVC) and sub-sensory threshold level and MVC and optimal stimulation level. A strong, significant Spearman correlation $\left(r_{s}=0.65\right)$ was observed between sub-sensory threshold level and optimal stimulation level. Although these correlations do not provide a means to predict optimal stimulation level as a function of these other measures, optimal stimulation level appears to increase with sub-sensory threshold and MVC.
\end{abstract}

Keywords: imperceptible, stimulation, vibrotactile, Gaussian noise, stochastic resonance, somatosensory system, sub-sensory threshold 


\section{Optimal Noise Level for Imperceptible Vibrotactile Stimulation}

\subsection{Introduction}

In recent decades, vibrotactile noise stimulation has received attention as a possible intervention to improve somatosensory function and supplement postural control (Priplata et al., 2002; Galica et al., 2009; Magalhaes \& Kohn, 2011; Lipsitz et al., 2015). Although the mechanism by which this intervention is effective is still uncertain, it has been theorized that mechanical noise stimulation elicits a stochastic resonance-like effect on the somatosensory system (Manjarrez et al., 2002; Collins et al., 2003; Moss \& Milton, 2003). Stochastic resonance (SR) is a naturally occurring phenomenon in which the detection of a signal in a non-linear system is improved with the application of random noise rather than degraded by it (Benzi et al.., 1981; Gammaitoni et al., 1995; McDonnell \& Abbott, 2009). Here, the somatosensory system serves as the non-linear system. The addition of noise may affect ion permeability of the mechanoreceptors (Bezrukov \& Vodyanoy, 1995) and may also sum constructively with the non-linear input signal increasing the likelihood that any near-threshold signal level will be positively detected (Moss et al., 2004; McDonnell \& Abbott, 2009). In other words, the application of vibrotactile noise may sharpen one's control of body position by providing additional sensory information and increasing the body's ability to detect and respond to changes in body orientation. Vibratory noise stimulation has shown to be effective in reducing postural sway among healthy young subjects as well as elderly subjects and patients with degraded somatosensory function due to diabetes and stroke (Collins et al., 2003; Priplata et al., 2002 \& 2006; Magalhaes \& Kohn, 2011). Additional work has demonstrated that postural sway may also be improved with other modes of noise stimulation. Sub-sensory electrical noise stimulation of the vestibular system via electrodes applied to the mastoid processes was shown to improve measures of head, trunk, and whole body stability during standing and walking tasks (Goel et al., 2015; Mulavara et al., 2015). As with vibrotactile stimulation, the electrical vestibular stimulation is thought to elicit an SR response within sensory systems governing balance.

Much of the previous work demonstrating the efficacy of vibrotactile noise stimulation has involved short-duration standing balance trials or isometric force production with the finger. This implies that the noise stimulation effect may be observed immediately without requiring a period of acclimation. While stabilizing effects have been observed for supra-sensory application of vibratory noise (Magalhaes \& Kohn, 2011), at least one study suggests that suprasensory applications may have a destabilizing effect due to the subject's awareness of it (Simeonov et al., 2011). Thus, the transparency of imperceptible stimulation and the immediacy of its effects make it an attractive intervention to aid stability rapidly and without distracting attention or otherwise disturbing the user.

Developing interventions which utilize imperceptible noise stimulation to aid with postural stability has been of interest for many years (Priplata et al., 2002; Collins et al., 2003; Magalhaes \& Kohn, 2011; Lipsitz et al., 2015). Currently, however, more research is needed regarding the signal content and amplitude that elicits the greatest benefits. In some cases, research groups have elicited postural stability improvements by applying imperceptible vibrotactile noise stimulation as a prescribed percentage (70-90\%) of an individual's sub-sensory threshold level (Priplata et al., 2002; Galica et al., 2009; Lipsitz et al., 2015). Despite observed stability improvements, uncertainties still exist regarding the mechanism responsible for these improvements. As discussed, it is frequently posited that improved stability with noise stimulation may be attributed to an SR-like effect within the somatosensory and somatomotor 


\section{Optimal Noise Level for Imperceptible Vibrotactile Stimulation}

systems. Previous research has suggested that there exists an optimal stimulation level which elicits greater performance improvements compared to stimulation at alternative levels (Manjarrez et al., 2002; Mendez-Balbuena et al., 2012; Trenado et al., 2014). There is an underlying assumption that supplemental stimulation to the somatosensory system should translate to improved somatomotor function. While somatosensory systems and somatomotor systems are integrated, they are not identical systems. Adhering to the principles of a stochastic system, it is not strictly necessary that optimal stimulation to one system should elicit optimal performance to a secondary system.

We aimed to further explore the concept of optimal vibrotactile noise stimulation in a directed motor control task. Specifically, we sought to quantify the optimal stimulation level for individuals being provided an identical but scaled white Gaussian noise $(\leq 500 \mathrm{~Hz})$ in a visual tracking finger flexion task. Participants completed a series of ramp-and-hold trials in which they attempted to maintain an isometric finger flexion force. During these trials, participants received imperceptible vibratory noise stimulation which was scaled to a specific percentage of the individual's sub-sensory threshold level. Task performance was quantified as a root-meansquare (RMS) error between the target and the generated isometric force values. Utilizing a consistent, scaled noise signal allowed us to investigate optimal stimulation level as a function of signal amplitude only. The goal of this study was to identify the optimal stimulation level for individuals and for a group of individuals via a directed motor control task and determine whether performance of a motor control task is significantly improved when receiving optimal sensory noise compared with other levels of sub-sensory stimulation. Additionally, correlations between optimal stimulation level, sub-sensory threshold, and/or maximal voluntary contraction capacities were identified to determine if optimal stimulation may be predictable based on another more easily quantified metric.

\subsection{Materials and methods}

The methods described here were part of a larger study conducted to explore the varying neurophysiological effects of vibrotactile noise stimulation during a simulated trigger pull task. As a component of that study, it was necessary to identify the subthreshold stimulation level which elicited the best force stability during a ramp-and-hold finger flexion task.

\subsection{Participants}

Previous work has demonstrated that motor neuron discharge characteristics and motor control are affected by sex differences (Tenan et al., 2016; Peng et al., 2018). For this study, only male subjects were recruited to avoid confounds due to sex differences. Eighteen (18) young male subjects (mean \pm standard deviation (SD): $25.8 \pm 6.2$ years of age) were recruited to participate in this study. Participants had no history of pain, surgery, or injury to the dominant upper extremity. Participants were also free from metabolic and neurological disorders, cardiovascular dysfunction, and did not take blood thinning medications. All methods were reviewed and approved by the Institutional Review Board of the U.S. Army Research Laboratory. Participants provided verbal and written informed consent prior to participation.

\subsection{Maximal Voluntary Contraction (MVC) procedures}

Participants were seated at an adjustable height desk equipped with a custom instrumented grip (Figure 1). The height of the desk was adjusted such that the participant could sit up straight 


\section{Optimal Noise Level for Imperceptible Vibrotactile Stimulation}

with their supine forearm resting flat on the surface of the desk. A custom adjustable cradle was mounted to the edge of the table to secure the position of the upper arm and help to standardize arm position between participants and between trials for the same participant (Figure 1A). The grip consisted of a plastic rod with a trigger-like mechanism instrumented with a compression load cell (LCM302-50, Omega Engineering, Inc., Stamford, CT). The angular orientation of the grip was adjustable to afford a comfortable neutral position for the wrist while the forearm and hand remained in supine position. The distance of the grip from the participant was adjusted such that the middle phalanx of the second finger engaged the trigger mechanism, and the remaining fingers curled comfortably around the plastic rod. All finger-pull tasks were completed with the hand and forearm in this standardized supine position.
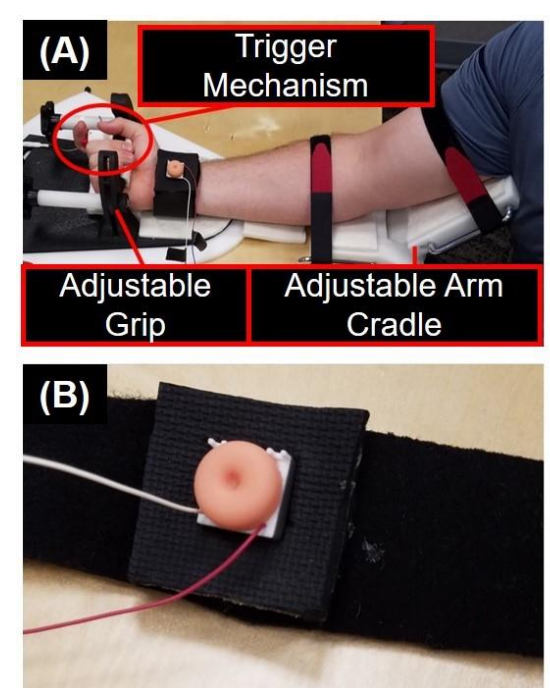

Figure 1. (A) A participant properly positioned and secured in the adjustable cradle and engaging the trigger-like mechanism. (B) The vibrotactile stimulator embedded in a neoprene wrist band. (C) A participant completing the ramp-and-hold task with visual feedback provided. (D) Magnified image of visual feedback indicating the target $20 \% \mathrm{MVC}$ level, the prompt to apply force and reach target level ('Ramp Up'), the duration of the force stabilization task, the middle 5 seconds of data used for the RMS error calculation, and the completion of the trial ('End').

Prior to completing other experimental tasks, participants completed a series of maximum exertions to determine their maximum finger flexion force. Participants were instructed to produce force only by squeezing their pointer finger against the trigger mechanism and were asked to refrain from flexing their wrist or bicep. A monitor positioned at the participant's eye level presented visual prompts and force feedback via custom data acquisition script (Spike2, Cambridge Electronic Design Ltd., Cambridge, England, UK). Verbal encouragement was also provided during maximum voluntary contractions (MVCs). MVCs were sustained for 5 seconds each with a rest period of 1 minute between successive attempts. Force data were amplified using a bridge amplifier (Digitimer NL109, Digitimer Ltd., Ft. Lauderdale, FL) and a gain of 100 and were recorded at a rate of $1000 \mathrm{~Hz}$ via a Micro 1401-3 data acquisition unit (Cambridge Electronic Design Limited, Cambridge, England, UK). After three exertions, the values for each MVC were analyzed. If the three consecutive MVC values were within $10 \%$ of each other, the highest MVC was recorded as the maximum force value. If there was more than a $10 \%$ 


\section{Optimal Noise Level for Imperceptible Vibrotactile Stimulation}

difference between the highest and lowest recorded MVC attempt, the participant was given 5 minutes of rest and another round of three MVCs were completed. This procedure continued until a maximum force value was identified. For all participants, this was accomplished in three or fewer rounds of MVC attempts.

Following the MVC procedures, each participant's individual sub-sensory threshold was determined. A vibrotactile stimulator (BM1C, Tactile Labs Inc., Montreal, Canada) was secured to the wrist of the participant's dominant hand using a neoprene strap (Figure 1B). All participants were right-hand dominant as determined by self-report. A white Gaussian noise signal $(\leq 500 \mathrm{~Hz})$ was generated with a custom Labview ${ }^{\mathrm{TM}}$ (v8.5, National Instruments Corp., Austin, TX) program. The signal was generated using Labview's Gaussian white noise VI with a standard deviation of the Gaussian probability density function equal to 2.0. The resulting signal was low-pass filtered at $500 \mathrm{~Hz}$, and ranged in amplitude from $+/-1.0$ with a mean of 0.0. This signal was scaled with two different gradations: $5-100 \%$ of full-scale in 5\% increments and $0.5-5 \%$ full scale in $0.5 \%$ increments. The second scaling gradation was necessary to provide a finer resolution for the identification of sensory threshold for participants' whose threshold was under 5\%. Similar white noise has been shown previously to improve performance of isometric force production tasks and includes the frequencies to which biological proprioceptors in the skin are sensitive (Trenado et al., 2014). The signals were transmitted to the wrist through the Micro 1401-3 unit via a custom Spike2 interface and delivered by the stimulator. Stimulation signals were presented for a duration of 5 seconds, and the magnitude of the signals and the time between successive stimulation signals was unknown to the participant. With eyes closed and hearing occluded with ear plugs, participants were asked to provide a "thumbs up" with their non-dominant hand when they detected stimulation. Researchers began by applying noise levels likely to be supra-sensory to confirm the participant understood instructions they were able to discern the vibratory stimulation. After approximately 5 trials of supra-sensory stimulation, the researcher applied a stimulation level which was expected to be on the cusp of perception. If the participant provided a positive response to the stimulation, the researcher followed by presenting the next 3 lower levels of noise stimulation. If the participant failed to provide a positive response to a stimulation, the researcher would apply noise two levels higher than that of the failed stimulation. The researcher would then proceed through the next 3 lower levels of noise stimulation. This process was repeated and afforded confirmation of threshold levels by ensuring that the participant would consistently report positive responses to levels above threshold and fail to detect other stimulation levels below threshold. The highest stimulation that the participant failed to detect three consecutive times was recorded as their sub-sensory threshold.

\subsection{Optimal noise stimulation protocol}

The optimal noise stimulation level was determined using a ramp-and-hold finger flexion task. Participants were presented with a visual display of the force applied to the trigger mechanism (Figure 1C). A visual cue would instruct the participant to gradually increase the force applied to the trigger over a period of 2.5 seconds to a maximum force of $20 \% \mathrm{MVC}$ and maintain this exertion for 10 seconds. Participants were instructed to maintain the $20 \%$ MVC as steadily as possible by tracing the visually presented $20 \%$ MVC target force with the force profile generated by finger flexion (Figure 1D). Ten practice trials were completed prior to data collection. To determine the optimal sub-sensory stimulation level, noise signals were generated corresponding 


\section{Optimal Noise Level for Imperceptible Vibrotactile Stimulation}

to $10-100 \%$ of their sub-sensory threshold. Participants completed 13 data trials in which they completed the ramp-and-hold task with or without stimulation applied to the wrist. The first and last trials were always sham trials in which no stimulation was applied. The sequence in which each noise signal (10 20,30, 40, 50, 60, 70, 80, 90, and 100\% sub-sensory threshold) was applied was randomized for each participant. Following the initial sham trial, a block of 5 rampand-hold trials were completed with different levels of stimulation. Participants then rested for five minutes. The first trial following the break was a sham trial, then 5 more ramp-and-hold trials were completed with the remaining stimulation levels followed by the final sham trial.

\subsection{Dependent measures}

Force stability, the primary measure of performance, was defined as the RMS error of the difference between actual force and the target force of $20 \%$ MVC. This value was calculated during the middle 5 seconds of the sustained MVC hold for each trial resulting in a total of 13 values per participant. The stimulation level corresponding to the lowest force stabilization RMS error (best performance) was recorded as the participant's optimal noise stimulation level.

\subsection{Statistical analyses}

RMS error data were analyzed at the group level using a multilevel model with stimulation level and subject as fixed and random effects, respectively. Post hoc statistical comparisons were made using Dunnett's test to correct for multiple comparisons (Dunnett, 1955). At the individual level, one-sided Dixon Q tests were used to examine whether performance during the identified optimal stimulation level was significantly different than the performance at all other levels of stimulation. Finally, Spearman correlations were used to identify whether relationships exist between threshold, MVC, and optimal stimulation values. All statistical analyses were conducted with R software (3.5.0, R Foundation for Statistical Computing).

\subsection{Results}

The MVC recorded for all participants ranged from $32.3 \mathrm{~N}$ to $188.2 \mathrm{~N}$ with a group mean $( \pm \mathrm{SD})$ of $93.2 \pm 39.2 \mathrm{~N}$. Input voltages identified as the sub-sensory threshold ranged from $0.02 \mathrm{~V}$ to $0.35 \mathrm{~V}$ with a group mean $( \pm \mathrm{SD})$ of $0.11 \pm 0.09 \mathrm{~V}$. This mean input voltage corresponds to an acceleration range of $0.064-0.275 \mathrm{G}$ across the frequency spectrum of the stimulation signals. Despite the wide range of input voltages identified as individual sub-sensory threshold levels, 9 participants $(50 \%)$ exhibited a sub-sensory threshold at an input voltage of $0.05 \mathrm{~V}(0.029-0.125$ $\mathrm{G}$ acceleration).

Force stabilization RMS errors recorded for each level of sub-sensory threshold stimulation varied greatly between participants (Figure 2). Multilevel model analyses were conducted with stimulation level as a fixed effect and subject as random intercept effect. No differences were observed between the RMS errors for the stimulation levels or the pre- or post-sham trials $\left(\mathrm{F}_{11,187}\right.$ $=1.45, \mathrm{p}=0.15)$. The largest RMS error magnitude $(0.54 \mathrm{~N})$ and standard error $(0.084 \mathrm{~N})$, however, occur for the final sham trial (post-sham) following the completion of noise stimulation trials. To examine whether this increase is due to a fatigue effect, a Pearson correlation was calculated between RMS error and the sequential trial number. No fatigue-related time-on-task effects were found $(r(16)=0.12, p>0.6)$. 


\section{Optimal Noise Level for Imperceptible Vibrotactile Stimulation}

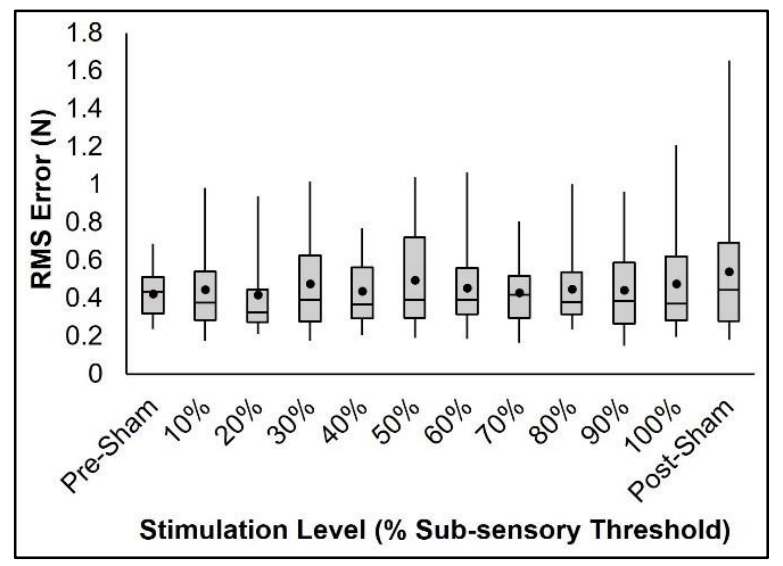

Figure 2. Box plots presenting $1^{\text {st }}$ quartile, median, and $3^{\text {rd }}$ quartile force stabilization RMS errors for each stimulation level. Top and bottom whiskers present the max and min RMS errors, respectively, for each stimulation level. The dots indicate the mean RMS error for each stimulation level.

Individual performance at each stimulation level varied greatly. Figure 3 presents the RMS errors recorded for a single participant exhibiting optimal stimulation at 30\% sub-sensory threshold.

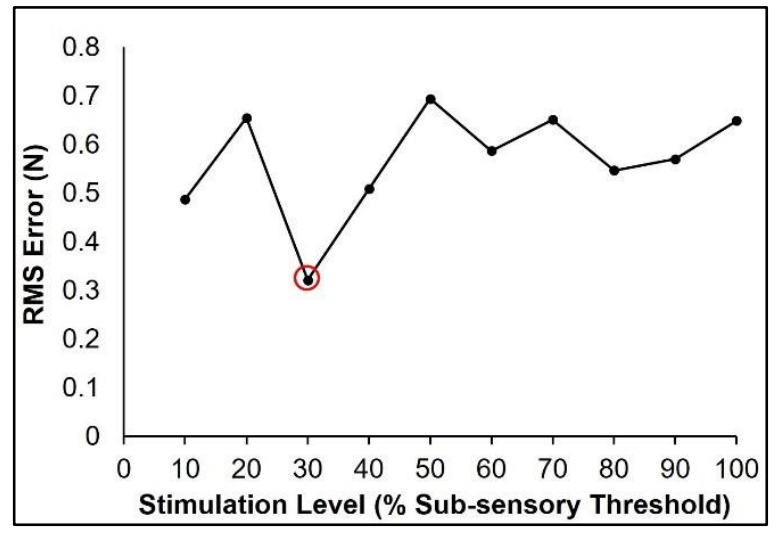

Figure 3. RMS errors for a single participant at each stimulation level. Optimal stimulation level was identified at $30 \%$ and is indicated by the circled data point.

To further examine the relationship between RMS errors and stimulation level for the group, the optimal stimulation level for each participant was aligned and associated error data was reclassified as a positive or negative percent difference (\%diff) from the optimal stimulation level. For example, for the data presented in Figure 4, the error at $30 \%$ sub-sensory threshold was identified as the optimal stimulation level, and the errors at 10, 20, 40, and 50\% were identified as $-20,-10,+10$, and $+20 \%$ diff, respectively, from the optimal stimulation level. Data for all participants were averaged for the optimal stimulation level and for each \%diff stimulation level (Figure 4). The result of this data presentation is that the number of values contributing to the mean and error calculations are different between stimulation levels. 
Optimal Noise Level for Imperceptible Vibrotactile Stimulation

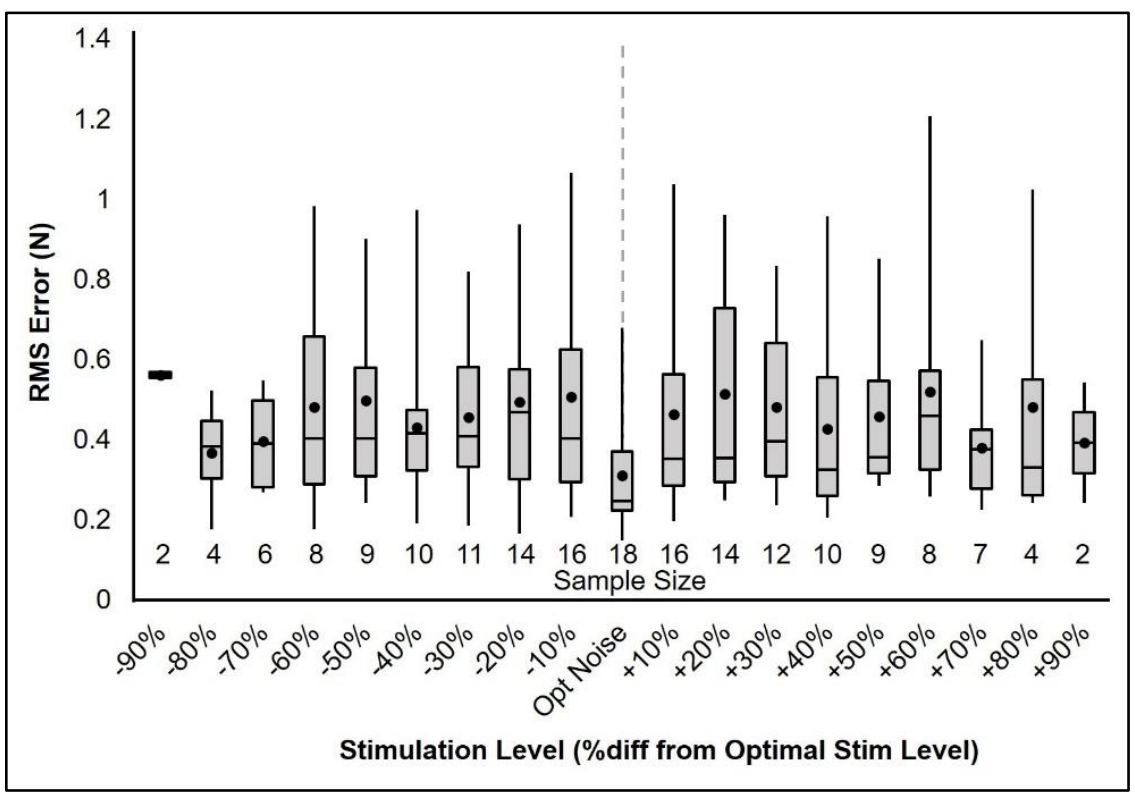

Figure 4. Box plots presenting $1^{\text {st }}$ quartile, median, and $3^{\text {rd }}$ quartile RMS errors for each stimulation level. Top and bottom whiskers present the maximum and minimum RMS errors for each level, respectively. The dots indicate the mean RMS error for each stimulation level. The number of observations used to determine mean, quartiles, maximum, and minimum values are provided as sample size corresponding to each stimulation level.

Model estimated marginal means, standard error, and confidence intervals are provided for each stimulation level in Table 1. When examining the RMS errors as a function of \%diff from optimal stimulation, the best performance as a group was observed at the optimal stimulation level. Multilevel analyses revealed that the model estimate RMS error recorded for the optimal stimulation level was significantly different $\left(F_{18,145}=3.06, p<0.0001\right)$ than the RMS errors for a number of stimulation levels using Dunnett's test (Dunnett, 1955) to correct for multiple comparisons. For stimulation levels ranging from $-60 \%$ to $+60 \%$, all but the $-30 \%$ level had $t$ scores ranging from $3.28 \leq \mathrm{t}_{145} \leq 5.46$ and significance levels of $\mathrm{p} \leq 0.019$. The stimulation level of $-30 \%$ fell just outside the cutoff for significance with $t_{145}=2.92, p=0.053$. Estimated RMS errors at stimulation levels of $-90 \%$ and $+80 \%$ were also significantly different than mean RMS error at the optimal stimulation level $\left(\mathrm{t}_{145}=3.08, \mathrm{p}=0.035\right.$ and $\mathrm{t}_{145}=3.61, \mathrm{p}=0.007$, respectively). In addition to the differences in estimated RMS values, the variation in performance as indicated by the box plot sizes also suggests that RMS errors at the optimal stimulation level had a narrower distribution than errors recorded for other stimulation levels. 
Optimal Noise Level for Imperceptible Vibrotactile Stimulation

Table 1. Estimated marginal means, standard errors, and confidence intervals (CI) from the multilevel statistical model.

\begin{tabular}{cccccc}
\hline $\begin{array}{c}\text { Stimulation } \\
\text { Level }\end{array}$ & $\begin{array}{c}\text { Estimated Marginal } \\
\text { Mean (N) }\end{array}$ & $\begin{array}{c}\text { Standard } \\
\text { Error (N) }\end{array}$ & $\mathrm{df}$ & $\begin{array}{c}\text { Lower } \\
95 \% \mathrm{CI}\end{array}$ & $\begin{array}{c}\text { Upper } \\
95 \% \mathrm{CI}\end{array}$ \\
\hline$-90 \%$ & 0.5475 & 0.0882 & 109.15 & 0.3726 & 0.7224 \\
$-80 \%$ & 0.4177 & 0.0714 & 64.09 & 0.2751 & 0.5603 \\
$-70 \%$ & 0.4352 & 0.0647 & 46.49 & 0.3050 & 0.5654 \\
$-60 \%$ & 0.4586 & 0.0610 & 37.81 & 0.3351 & 0.5822 \\
$-50 \%$ & 0.4850 & 0.0597 & 34.96 & 0.3638 & 0.6063 \\
$-40 \%$ & 0.4410 & 0.0586 & 32.68 & 0.3217 & 0.5603 \\
$-30 \%$ & 0.4229 & 0.0577 & 30.83 & 0.3051 & 0.5406 \\
$-20 \%$ & 0.4844 & 0.0557 & 27.02 & 0.3701 & 0.5988 \\
$-10 \%$ & 0.4955 & 0.0548 & 25.37 & 0.3828 & 0.6084 \\
Opt Noise & 0.3106 & 0.0541 & 24.14 & 0.1989 & 0.4222 \\
$+10 \%$ & 0.4653 & 0.0548 & 25.37 & 0.3525 & 0.5781 \\
$+20 \%$ & 0.4992 & 0.0557 & 27.02 & 0.3849 & 0.6136 \\
$+30 \%$ & 0.4634 & 0.0569 & 29.32 & 0.3470 & 0.5798 \\
$+40 \%$ & 0.4467 & 0.0586 & 32.67 & 0.3274 & 0.5660 \\
$+50 \%$ & 0.4706 & 0.0597 & 34.96 & 0.3493 & 0.5918 \\
$+60 \%$ & 0.5083 & 0.0610 & 37.82 & 0.3847 & 0.6318 \\
$+70 \%$ & 0.4309 & 0.0626 & 41.50 & 0.3045 & 0.5773 \\
$+80 \%$ & 0.5160 & 0.0713 & 63.98 & 0.3735 & 0.6585 \\
$+90 \%$ & 0.4831 & 0.0882 & 109.07 & 0.3082 & 0.6579 \\
\hline
\end{tabular}

Individual one-sided Dixon Q tests were conducted to determine whether the minimum RMS error recorded for a given participant could be identified as significantly different from the group of RMS errors recorded for other stimulation levels. Two participants (participants 4 and 11) demonstrated RMS errors that were significantly lower $(Q=0.50, p=0.039$ and $Q=0.54, p=$ 0.024 , respectively) than the remaining distribution of errors recorded for the other stimulation levels. To further inspect the distribution of errors for each participant, parametric $95 \%$ confidence intervals were calculated for each individual (Figure 5). The difference between a participant's lowest RMS error and their lower 95\% confidence limit ranged from $0.005 \mathrm{~N}-0.21$ $\mathrm{N}$ with a group mean ( $\pm \mathrm{SD}$ ) difference of $0.08 \pm 0.06 \mathrm{~N}$. No consistency was observed in the sub-sensory stimulation level eliciting the best force stabilization (Figure 5). Among the 18 participants, each sub-sensory stimulation level was found to be optimal for at least one individual (Figure 5). 
Optimal Noise Level for Imperceptible Vibrotactile Stimulation

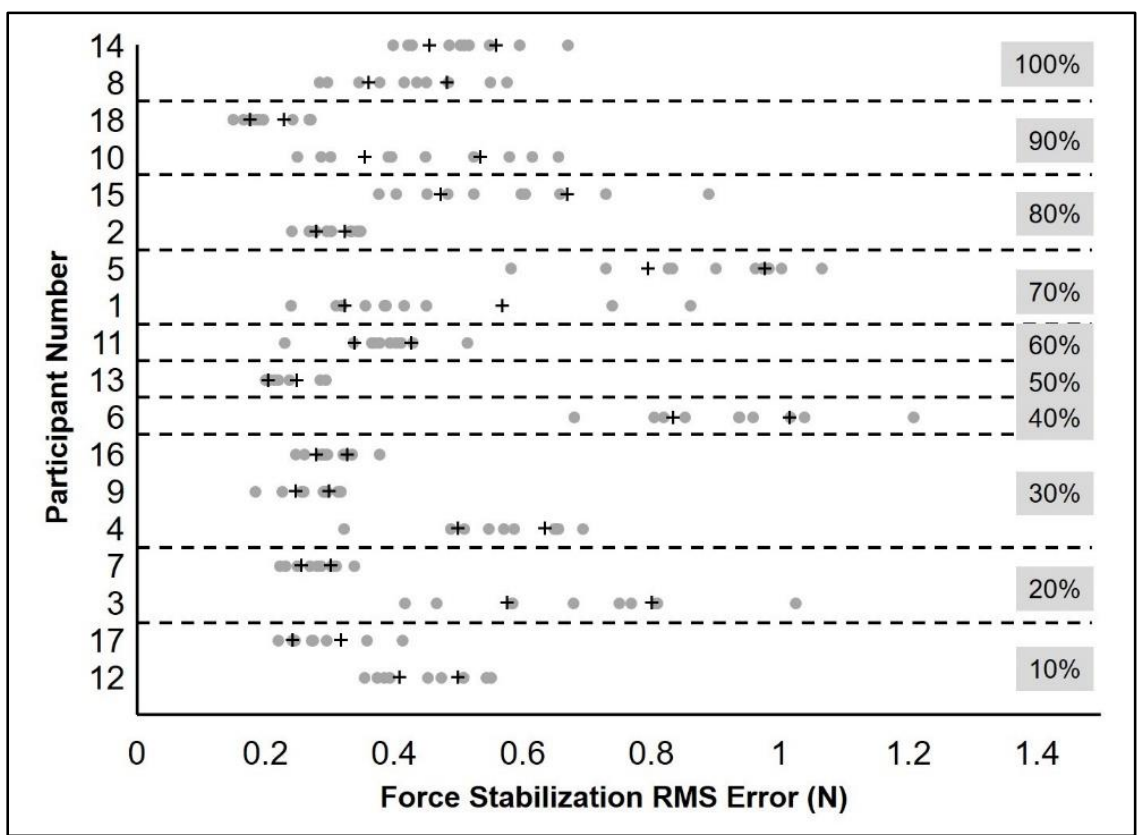

Figure 5. RMS errors recorded for each of 10 stimulation levels. The symbol "+" for each data set indicates the upper and lower limits of the $95 \%$ confidence interval. Individual subject data is presented and grouped by optimal stimulation level. Shaded values on the right indicate the optimal stimulation level as a percent of individual sub-sensory threshold.

Following the force stabilization trials, the input voltages identified as the mean optimal noise stimulation level had a range of $0.005-0.16 \mathrm{~V}$ with a group mean $( \pm \mathrm{SD})$ of $0.06 \pm 0.05 \mathrm{~V}$. This mean optimal stimulation voltage corresponded to acceleration magnitudes of $0.035-0.15 \mathrm{G}$ across the frequency spectrum of the stimulation signals. To examine whether significant relationships exist between threshold, optimal stimulation level, and MVC values, Spearman correlations were calculated for each pair of measures. Spearman, rather than Pearson, correlations were used due to the lack of normality for the threshold and optimal stimulation level measures as determined by the Shapiro-Wilk test $(\mathrm{W}=0.80, \mathrm{p}=0.002$ and $\mathrm{W}=0.83, \mathrm{p}=$ 0.005, respectively). Moderate but significant Spearman correlations were observed between threshold and MVC $\left(\mathrm{r}_{\mathrm{s}}=0.49, \mathrm{p}=0.041\right)$ and optimal stimulation level and $\mathrm{MVC}\left(\mathrm{r}_{\mathrm{s}}=0.56, \mathrm{p}=\right.$ 0.016). Increases in MVC are associated with increases in both threshold and optimal stimulation level. A strong significant Spearman correlation was observed between threshold level and optimal stimulation level $\left(\mathrm{r}_{\mathrm{s}}=0.65, \mathrm{p}=0.003\right)$.

\subsection{Discussion}

In general, group level performance of a finger force stability task was significantly improved when participants received stimulation corresponding to their individually identified optimal stimulation level. When performance was assessed at an individual level, however, RMS errors during optimal stimulation trials were only significantly different from the distribution of errors across other noise stimulation trials for 2 participants. Noise stimulation appears to have a positive effect on force stability, but it may be unnecessary to identify an optimal stimulation level at the individual level. Optimal stimulation levels are highly variable, and this data 


\section{Optimal Noise Level for Imperceptible Vibrotactile Stimulation}

suggests that the performance improvement compared with other levels of stimulation are marginal for most individuals.

Despite a broad range in MVC measures (32 to $188 \mathrm{~N}$ ) and sub-sensory thresholds (0.02 to $0.35 \mathrm{~V}$ ), half of the participants ( 9 of 18) exhibited their sub-sensory threshold level at $0.05 \mathrm{~V}$. The reason for the cluster of threshold levels at $0.05 \mathrm{~V}$, however, is unclear. Noise signals were evaluated for threshold in increments of $0.05 \mathrm{~V}$. The next highest noise magnitude was $0.10 \mathrm{~V}$. It may be that the acceleration magnitudes imparted by the stimulator between input voltages of 0.10 and $0.05 \mathrm{~V}$ are very near the central distribution of perception. The $0.05 \mathrm{~V}$ step between these two input voltages may have been too coarse to specifically pinpoint the input voltage level at which sensation ceases. Future studies may utilize a finer steps between input voltages to identify sub-sensory threshold levels.

Individual performance during the ramp-and-hold task with a specific noise stimulation level varied substantially. The variability was so large that, when averaged among all participants, the presence of any group-level optimal stimulation level was obscured. Similar findings were reported by Manjarrez et al. (2002) who noted considerable variability in the shape of electroencephalography signal-to-noise ratio profiles obtained for different participants receiving the same input noise levels. Individual responses to noise stimulation appear to be highly variable and are not easily or effectively described via standard summary statistics. Mulavara et al. (2015) found similar results with electrical vestibular stimulation. Although they reported a group average optimal stimulation level equivalent to $35 \%$ of perceptual threshold, individuals exhibited optimal stimulation levels ranging from $8-100 \%$ of perceptual threshold. Additionally, using electrical vestibular stimulation, Goel et al. (2015) reported that during each of 4 studies, only a subset of participants were considered "responsive" to stimulation. Large inter-subject variability and the existence of "non-responders" to stimulation make the study of optimal noise stimulation levels challenging. Of note in the present study, however, is that the largest RMS error and largest variability occurred for the final sham trial. This suggests that noise stimulation, regardless of its level, may have imparted some degree of stabilization in the preceding trials, and its absence during the final sham trial may have resulted in poorer performance across participants. This finding raises a concern not often addressed in the noise stimulation literature. It may be relevant to quantify the effects of sudden cessation of noise stimulation, particularly for noise stimulation interventions.

Two different attempts were made to determine if a true optimal level of stimulation exists to increase stability. The first analysis performed a partial pooling of all subject's data using a multilevel model with the data restructured and centered at each individual's optimal stimulation level. This analysis revealed that there did appear to be a statistical difference between the level of stimulation eliciting the greatest stability and other levels of stimulation. These results support previous research indicating that there is an optimal noise level for enhanced motor performance (Manjarrez et al., 2002; Mendez-Balbuena et al, 2012). The second analysis considered individual subject data and attempted to determine if the behavioral outcome (i.e. RMSE stability) from the optimal stimulation level was a statistical outlier compared to all other trials. These results failed to show that the optimal stimulation level was clearly beneficial in most of the subjects. For only two participants was the RMS error during their optimal noise trial significantly lower than the error during other trials. For these individuals, an optimal 
Optimal Noise Level for Imperceptible Vibrotactile Stimulation

stimulation level appears to have produced better motor performance. It is possible that not enough data points were collected to effectively determine if stability during the optimal stimulation level resides outside the expected distribution of data. Indeed, this is one of the known strengths of the partial pooling of data within a multilevel model framework. Future research in this area should obtain data at more points along the continuum of stimulation or perform repeated testing on the same stimulation levels to confirm the optimal noise hypothesis for motor behavior.

Optimal stimulation levels resulted in force stabilization RMS errors which fell outside the 95\% CI for all subjects. As reported previously (Manjarrez et al., 2002; Mendez-Balbuena et al., 2012), the optimal stimulation level for individuals varied widely, and RMS error curves exhibited a variety of shapes. Manjarrez et al. (2002) arbitrarily defined 3 bands of noise and classified their participants as responders to either low, intermediate, or high levels of noise stimulation. The majority of their participants exhibited optimal performance within the high range of noise stimulation. Using the same technique, we would define our low, intermediate, and high noise levels as bands consisting of 10-30\%, 40-70\%, and 80-100\%, respectively. Optimal performance in our study, however, was more evenly distributed among the three identified bands of noise with 7 participants exhibiting optimal noise stimulation in the low band, 5 in the intermediate band, and the remaining 6 in the high band. Currently, research employing vibratory noise stimulation often applies noise at a specific percentage ( 70-90\%) of sub-sensory threshold level (Priplata et al., 2002; Galica et al., 2009; Lipsitz et al., 2015). Results from these studies clearly indicate that this arbitrary noise level does elicit a stabilizing effect compared to no stimulation. However, this commonly used stimulation level may actually only be eliciting the optimal stabilization effects for about $1 / 3$ of the participants. Greater stabilization may be possible if optimal stimulation levels can be identified and applied; however, this level of customization may be unnecessary in some motor tasks or provide only marginal benefits over an arbitrary stimulation level.

Studies using designs similar to ours have indicated that an optimal level of noise is required to obtain a maximal effect of noise stimulation (Manjarrez et al., 2002; Mendez-Balbuena et al., 2012; Trenado et al., 2014). Our results, however, do not exhibit a robust optimal noise phenomenon with a predictable inverted-U pattern. While the optimal noise pattern is a classical hallmark of stochastic resonance within a non-linear system, the present study focused on two different systems: the somatosensory and the motor systems. It is possible that the SR-like benefit in the somatosensory system does not translate directly into the motor system, which is receiving numerous neurological inputs to generate force. The two previous study designs showing an inverted-U optimal noise pattern were also focused on fingertip displacement (Manjarrez et al., 2002; Mendez-Balbuena et al., 2012). It is possible that some characteristics of noise stimulation on motor behavior are only able to be differentiated within specific paradigms and not widely generalizable to other tasks.

As a final quantitative analysis of the present data, Spearman correlation coefficients were calculated for MVC, sub-sensory stimulation thresholds, and optimal noise stimulation level. Significant moderate to strong correlations were found suggesting that a higher MVC and a higher sub-sensory threshold level are associated with higher optimal stimulation levels. A larger MVC was also associated with a higher sensory threshold. These relationships are 
Optimal Noise Level for Imperceptible Vibrotactile Stimulation

relevant when customizing stimulation. Individuals with a larger MVC magnitude likely warrant a greater stimulation magnitude for customized noise stimulation. However, the quantitative relationship between optimal noise stimulation, sub-sensory threshold level, and finger flexion MVC was not identified here. Simplifying the selection of optimal noise stimulation level would expedite its use in research and in clinical applications. More research is needed to determine if in fact an optimal stimulation level is essential for clinical interventions and, if so, whether an individual's optimal stimulation level may be effectively identified via a more easily quantified measure.

\subsection{Limitations}

A primary limitation of this study was the completion of only a single trial for each noise stimulation level. While other studies have been conducted in a similar fashion (Manjarrez et al., 2002; Mendez-Balbuena et al., 2012; Trenado et al., 2014), collecting additional trials would have provided a measure of performance variability for each noise level and afforded reliability analyses for the optimal stimulation level. Additionally, there is some uncertainty regarding the duration of the effects of stimulation. For this study, participants received a particular level of stimulation for the duration of a given trial. It is unclear whether exposure time to imperceptible stimulation has an influence on its behavioral effects.

\subsection{Conclusions}

Despite tightly controlling other signal properties and varying only noise amplitude, the optimal stimulation level varied considerably between individuals. The lack of significant performance differences at the individual level between optimal stimulation levels and the remaining trials may indicate that, while optimal stimulation provides the best performance, its benefits may only marginally outweigh noise stimulation at an arbitrary imperceptible level when focusing on fingertip displacement. Additional work is needed to determine the reliability of the optimal noise stimulation levels identified using this procedure. Developing a reliable method of identifying an individual's optimal noise stimulation level would support the development of customizable stability aids or interventions to provide maximal assistance to the user.

\subsection{Acknowledgements}

Many thanks to Mr. Mike Kosinski for his hard work designing and fabricating the instrumented grip apparatus and the arm cradle used for data collection. Thanks also to Dr. J. Cortney Bradford and the facilities staff at the Army Research Laboratory Mission Impact through Neuro-inspired Design (MIND) lab for the use of their equipment and lab facilities.

\section{Conflict of Interest Statement}

The authors declare that this research was conducted independently and free of any personal or financial relationships that could be construed as a conflict of interest.

\section{Funding}

This work was funded by the Department of Defense Human Systems Integration (Cybernetics) research line at the U.S. Army Research Laboratory (74-A-HRCYB). 


\section{Optimal Noise Level for Imperceptible Vibrotactile Stimulation}

\section{Author Contributions Statement}

$\mathrm{CH}$ - Dr. Haynes assisted with all data collection sessions, processed and analyzed the present data set, generated the graphs and images, and drafted the full manuscript.

MT - Dr. Tenan assisted with all data collection sessions, conducted statistical analyses for the present data set, and provided editorial revisions of the manuscript.

AP - Dr. Passaro assisted with developing the testing procedures, advised on the analyses and data presentation, and provided editorial revisions for the manuscript.

AT - Mr. Tweedell assisted with all data collection sessions and provided editorial revisions of the manuscript.

\section{References}

Benzi, R., et al. (1981). "The mechanism of stochastic resonance." J Phys A: Math Gen 14(11): L453-L457.

Bezrukov SM, Vodyanoy I. Noise-induced enhancement of signal transduction across voltage dependent ion channels. Nature (1995) 378: 362-364.

Collins JJ, Priplata AA, Gravelle DC, Niemi J, Harry J, Lipsitz LA. Noise-enhanced human sensorimotor function. IEEE Eng Med Biol (2003) Mar/Apr: 76-83.

Dunnett CW. A multiple comparison procedure for comparing several treatments with a control. J Am Stat Assoc (1955) 50(272): 1096-1121.

Galica AM, Kang HG, Priplata AA, D'Andrea SE, Starobinets OV, Sorond FA et al. Subsensory vibrations to the feet reduce gait variability in elderly fallers. Gait \& Post (2009) 30: 383387.

Gammaitoni L, Hanggi P, Jung P, Marchesoni F. Stochastic resonance. Rev Mod Phys (1998) 70(1): 2233-287.

Goel R, Kofman I, Jeevarajan J, De Dios Y, Cohen H, Bloomberg JJ et al. Using low levels of stochastic vestibular stimulation to improve balance function. PLoS ONE (2015) 10(8):e0136335. Doi:10.1371/journal.pone.0136335.

Lipsitz LA, Lough M, Niemi J, Travison T, Howlett H, Manor B. A shoe insole delivering subsensory vibratory noise improves balance and gait in healthy elderly people. Arch Phys Med Rehabil (2015) 96(3): 432-439.

Magalhaes FH, Kohn AF. Vibratory noise to the fingertip enhances balance improvement associated with light touch. Exp Brain Res (2011) 209: 139-151. 


\section{Optimal Noise Level for Imperceptible Vibrotactile Stimulation}

Manjarrez E, Diez-Martinez O, Mendez I, Flores A. Stochastic resonance in human electroencephalographic activity elicited by mechanical tactile stimuli. Neurosci Lett (2002) 324: 213-216.

Mendez-Balbuena I, Manjarrez E, Schulte-Monting J, Huethe F, Tapia JA, Hepp-Raymond M-C, et al. Improved sensorimotor performance via stochastic resonance. J Neurosci (2012) 32(36): 12612-12618.

McDonnell MD, Abbott D. What is stochastic resonance? Definitions, misconceptions, debates, and its relevance to biology. PLOS Comput Biol (2009) 5(5): 1-6.

Moss F, Milton JG. Balancing the unbalanced. Nature (2003) 425: 911-912.

Moss F, Ward LM, Sannita WG. Stochastic resonance and sensory information processing: a tutorial and review of application. Clin Neurophysiol (2004) 115: 267-281.

Mulavara AP, Kofman IS, De Dios YE, Miller C, Peters BT, Goel R et al. Using low levels of stochastic vestibular stimulation to improve locomotor stability. Front Syst Neurosci (2015) 9:117.

Peng Y-L, Tenan MS, Griffin L. Hip position and sex differences in motor unit firing patterns of the vastus medialis and vastus medialis oblique in healthy individuals. J Appl Physiol (2018) 124: 1438-1446.Priplata AA, Niemi J, Salen M, Harry J, Lipsitz LA, Collins JJ. Noiseenhanced human balance control. Phys Rev Lett (2002) 89(23): 238101-1-238101-4.

Priplata AA, Patritti BL, Niemi JB, Hughes R, Gravelle DC, Lipsitz LA, et al. Noise-enhanced balance control in patients with diabetes and patients with stroke. Ann Neurol (2006) 59:412.

Severini G, Delahunt E. Effect of noise stimulation below and above sensory threshold on postural sway during a mildly challenging balance task. Gait \& Post (2018) 63: 27-32.

Simeonov P, Hsiao H, Powers J, Ammons D, Kau T, Amendola A. Postural stability effects of random vibration at the feet of construction workers in simulated elevation. Appl Ergo (2011) 42: 672-681.

Tenan MS, Hackney AC, Griffin L. Maximal force and tremor changes across the menstrual cycle. Eur J Appl Physiol (2016) 116: 153-160.

Trenado C, Mikulic A, Manjarrez E, Mendez-Balbuena I, Schulte-Monting J, Huethe F, et al. Broad-band Gaussian noise is most effective in improving motor performance and is most pleasant. Front Hum Neurosci (2014) 8(22): 1-9. 